\title{
La colección digital de cuadernos de historia en la educación básica: una fuente documental para la investigación de las prácticas escolares en México
}

Dra. María Guadalupe Mendoza Ramírez, Mexicana.

ORCID 0000-0003-4895-1641

mmendozaupntoluca@gmail.com

Dr. Rodolfo Cruz Bustos, Mexicano.

ID ORCID 0000-0002-7260-7122

rcbustos@gmail.com

Mtro. Rodolfo Huerta González, Mexicano.

ID ORCID 0000-0002-4623-8087

rhuerta12@gmail.com

Universidad Pedagógica Nacional, Unidad 151-Toluca

Recibido: 14 de septiembre del 2020

Aceptado: 10 de octubre del 2020

\section{Resumen}

Este trabajo de investigación educativa sistematiza el proceso de conformación de la Colección Digital de Cuadernos Escolares de Historia (CESHIS), especializada en Historia de México e Historia Universal, coordinada por un equipo de docentes e investigadores de la Universidad Pedagógica Nacional. En este artículo se describen las fases de búsqueda, digitalización y análisis de una cohorte de cuadernos de Historia de México e Historia Universal producto de la reforma integral de educación básica (2011-2016). Se presenta el diseño metodológico de la colección de la colección Cuadernos CESHIS y del contenido de la base de datos especializada. Los resultados de esta investigación sobre producciones escolares, invita a los estudiosos de la cultura escrita a generar una nueva mirada a la escuela, sus producciones infantiles y las prácticas de escritura en la enseñanza de la historia.

Palabras clave: Enseñanza de la historia, Educación básica, investigación. 


\title{
The digital collection of history notebooks in basic education: a documentary source for research on school practices in Mexico
}

\begin{abstract}
This educational research work systematizes the process of conformation of the Digital Collection of History School Notebooks (CESHIS), specialized in Mexican History and Universal History, coordinated by a team of teachers and researchers from the National Pedagogical University. This article describes the search, digitization and analysis phases of a cohort of notebooks on the History of Mexico and Universal History as a result of the comprehensive reform of basic education (2011-2016). The methodological design of the CESHIS collection and the content of the specialized database are presented. The results of this research on school productions invites students of written culture to generate a new look at school, its children's productions and writing practices in the teaching of history.
\end{abstract}

Keywords: History teaching, basic education, research.

\section{Introducción}

Los cuadernos escolares son una fuente inagotable para documentar los procesos de enseñanza de las disciplinas escolares en diferentes contextos y momentos curriculares al abrir la ventana a las prácticas de escritura de la historia que los niños y jóvenes despliegan en las aulas. La historia es actualmente una asignatura poco valorada pues las reformas educativas en México le han dado prioridad a las matemáticas y a la enseñanza de la lengua.

El cuaderno escolar de historia es una fuente de difícil acceso y conservación; por ello esta colección es pionera en la investigación de cuadernos escolares en México. En este trabajo presentamos el sustento metodológico y las etapas por las que pasó el proceso de acopio, el diseño, la digitalización y el análisis de los cuadernos que conforman esta colección que constituye un riquísimo insumo de investigación de la historia reciente de la educación histórica en México.

Los cuadernos escolares son una fuente histórica y socioeducativa que permite reconstruir, analizar y valorar las prácticas en la enseñanza de las disciplinas como la historia; 
dilucidar las intenciones pedagógicas de la intervención docente y las prácticas de escritura y de lectura asociadas al uso de los libros de texto en el aula y a los procesos de enseñanza y el aprendizaje de la historia escolar.

Para los historiadores los cuadernos escolares entrecruzan tres caminos: la historia de la infancia, la historia de la educación (de la cultura escolar) y la cultura escrita. Los cuadernos escolares, como fuente documental compleja, tienen el privilegio de ser un producto nacido en la escuela lo que permiten acercarnos a la "intrahistoria" de la escuela. En los estudios sobre los cuadernos escolares en España y América Latina se identifican dos vertientes de investigación: la pedagógica y la histórica, que consideran a los cuadernos como objeto de estudio y como fuente histórica respectivamente.

La primera línea considera al cuaderno escolar como un medio, como un soporte físico que posibilita la observación de la problemática referida a los contenidos escolares; se trabaja sobre los temas contenidos en los cuadernos y la forma en que son tratados. Desde esta vertiente cobra importancia el análisis de la textualidad del cuaderno; la escritura y los rasgos que adquieren los "contenido aprehendidos" en la fase de recepción del texto y reelaboración del contenido. El cuaderno, como objeto de estudio, contiene los elementos pedagógicos y/o historiográficos de una cadena que inicia en el programa de estudios, pasa por la planeación docente, el uso del libro de texto y finalmente se plasma en un contenido reelaborado por el alumno.

Esta dimensión de análisis es lo que Anne-Marie Chartier denomina, metafóricamente, "la vitrina de la clase" (2005:31) aportando nuevos elementos desde la perspectiva metodológica de la cultura escrita. Desde esta mirada, se considera al cuaderno de clase, como un soporte físico, un espacio en el que queda registrado gran parte de la labor cotidiana realizada en el aula, pero también como un dispositivo escolar, que conforma prácticas discursivas orales y escritas (Chartier, 2005:34). El cuaderno y la carpeta escolar, se consideran fuentes para el análisis e interpretación de la realidad diaria tanto en el aula como de las prácticas que quedan registradas en los cuadernos y/o carpetas, en el modo de transmitir los contenidos y de organizar las actividades de acuerdo a los estilos docentes. 
La segunda perspectiva de investigación considera al cuaderno escolar como fuente para documentar la vida escolar, en tanto que es un recurso auxiliar del proceso de enseñanza - aprendizaje; y constituye un registro escrito de todas las actividades que se realizan en el aula cotidianamente y como un mediador entre la escuela, la familia y el niño. Los estudios de A. Viñao (2006) y A. Escolano (1997) sostienen que los cuadernos escolares son un objeto fuertemente normado con un uso totalmente ritualizado que lo convierte en un instrumento de control social. Dentro de esta otra corriente, se insertan los estudios de cultura escrita y cultura escolar que toman al cuaderno como un instrumento didáctico, dado que su estructura contiene "lo enseñado" y el conocimiento del alumno cuyo valor es evaluado a partir de premios y castigos (Viñao, 2007:10).

Los cuadernos como fuente y objeto de investigación en la Historia de la Educación tienen una trayectoria que data de aproximadamente dos décadas, Sanchidrián \& Arias (2013) hacen un recuento de los estudios históricos de los cuadernos escolares como parte de la cultura material de la educación. Las investigaciones españolas e italianas, principalmente, se han dado a la tarea de recuperar y sistematizar esta fuente mediante la elaboración de bases de datos y exposiciones de cuadernos escolares y museos pedagógicos. Para la Historia de la Educación el cuaderno escolar nos remite y acerca a la realidad educativa desde distintas perspectivas en base a las diversas concepciones y modos de interpretar que tenemos de la realidad social.

Los cuadernos escolares, como producto de la cultura material, representan el sistema político y moralista de la época seleccionada. La cultura empírica de la enseñanza y la experiencia nos ha legado un conjunto de voces, escrituras, íconos y objetos que son expresión de la cultura material de la escuela, en las que hay que posar la mirada etnográfica y micro-histórica para aprender el valor de la memoria pedagógica, de sus prácticas y sus discursos, de sus tradiciones y de sus virtualidades.

\section{Antecedentes}

Las colecciones de cuadernos escolares son un campo fértil de la investigación de la historia escolar. En 2005 el seminario interdisciplinar de Estudios sobre Cultura Escrita (SIECE) de la Universidad el Seminario Interdisciplinar de Estudios sobre Cultura Escrita de la 
Universidad de Alcalá (SIECE) realizó diversas actividades científicas y de formación que en su conjunto dieron lugar al primer año de funcionamiento como grupo investigador en el seno del Departamento de Historia I y Filosofía de la Universidad de Alcalá (Madrid). Los cuadernos escolares como fuente y como objeto de investigación se empezaron a documentar en los encuentros, congresos de cultura escrita y proyectos de investigación conjuntos.

En el cuarto y quinto encuentro del seminario interdisciplinar de la cultura escrita en 2004-2005, los proyectos de acopio y sistematización de bases de datos sobre libros escolares y la cultura escrita desde la Educación que habían iniciado años antes los historiadores R. Chartier y A. Viñao Frago. También en el contexto español, Benito Escolano consolida y constituye un centro reproductor de proyectos de acopio y sistematización de libros y cuadernos escolares en Europa.

En 2007 se llevó a cabo en Macerata, Italia, el primer Congreso Internacional sobre los cuadernos escolares (Sanchidrián \& Gallego, 2003) y tan importante fue la presencia del estudio de los cuadernos escolares como objetos museológicos, que en 2008 en Huesca, España, la Sociedad del Patrimonio Histórico Educativo (SEPHE) organizó su tercer congreso internacional. Paralelamente en América Latina se incentivaron las investigaciones de la cultura escrita a partir de los congresos internacionales de cuadernos escolares que se llevaron a cabo en Colombia y Argentina donde se constituyeron equipos de investigación que dieron origen a la presentación de ponencias especializadas.

Será finalmente la tradición francesa de historia de la educación, a través de la Universidad de Limoges (Francia) la primera institución en crear una magnífica colección digital de cuadernos escolares. Esta colección se conserva en el INSPE de la Academia de Limoges, principalmente en el sitio de Limoges Condorcet. Dentro de este fondo patrimonial, hay una amplia colección tanto cuadernos de estudiantes como de cuadernos del maestro. Los primeros catálogos de la colección permitieron contar más de 400 cuadernos, formados por donaciones de antiguos alumnos o ex maestros, la más antigua de las cuales data de 1871.

Esta importante colección consta de cuadernos de estudiantes de la escuela primaria posterior a 1920 además de los libros de maestros y estudiantes de secundaria. Entre estos 
cuadernos de estudiantes, se enumeran varios tipos, entre otros: cuadernos disciplinarios, cuadernos, cuadernos colectivos, cuadernos, cuadernos mensuales, cuadernos diarios, talonarios y cuadernos de borradores. En cuanto al contenido, todas las disciplinas de la escuela están representadas: moral, recitación, aritmética, vocabulario, escritura, historia, lectura, canto, geometría, ortografía, geografía, gimnasia, ciencias, métricas, escritura, etc.

La catalogación y digitalización de esta colección se llevó a cabo, bajo la responsabilidad de Marc Moyon (INSPE de la Academia de Limoges), gracias al proyecto de cooperación internacional "La enseñanza de las matemáticas en la escuela primaria, siglos XIX-XX: estudios comparativos, Brasil-Francia". Esta valiosa documentación y accesibilidad para toda investigación en historia de la educación o la didáctica. Esta base de datos se enriquecer progresivamente con archivos escaneados.

\section{Metodología}

El cuaderno de Historia no es un reflejo de las normas y principios pedagógicos y curriculares que subyacen en la escuela, sino que constituye un dispositivo que construye, reproduce, o modifica las prácticas escolares y en sí misma da cuenta de varias dimensiones de análisis: las que se centran en la forma que adquiere el contenido enseñado, las prácticas de lectura de los infantes, los signos y significados de la escritura de la historia en el aula y sobre todo la posibilidad o limitación de la escritura autónoma.

Las prácticas discursivas escolares son el discurso escolar y su propia trama "las mutaciones que podemos encontrar en los cuadernos escolares (de clase) no están relacionadas directamente con ciclos de políticas educativas o con la evolución de las disciplinas científicas, sino que parecen obedecer a otras vicisitudes propias de la construcción discursiva y del devenir de la institución escolar" (Carretero, 2007). Devenir que está determinado sólo en su parte más visible y de manera vaga por dichas políticas, y que obedece a conocimientos, concepciones y a una gramática escolares específicas que tienen una lógica propia.

Del lado del profesor, los cuadernos de Historia pueden reflejar, entre otras cosas, su concepción de los sujetos que la hacen (personajes, políticos, presidentes u otros actores sociales); la naturaleza de la historia (lo que ya pasó, o tiene relación con el presente, o tiene 
una interacción del pasado no solo con el presente sino también con el futuro); las funciones intelectuales que le da a la historia como las figurativas (operaciones mentales que no tienden a transformar al objeto sino a proveer una reproducción informativa) o las operativas (donde la información transforma realmente la idea de la historia); y las estrategias de enseñanza que más utiliza (resúmenes, cuestionarios, mapas históricos, líneas del tiempo).

Del lado del alumno, este material puede mostrar si el alumno está orientado al aprendizaje significativo o mecánico y, por lo tanto, que significación le está dando al conocimiento historia; su nivel de comprensión de los hechos históricos (acumulación de datos, relaciones de causalidad, interpretación o comprensión de la historia); si desarrolla pensamiento y conciencia históricas; si visualiza la historia en relación a su entidad federativa, municipio, comunidad, familia e historia personal; su juicio personal acerca de los hechos históricas y hasta sus propias actitudes, sentimientos y emociones con respecto a la historia.

Desde estos supuestos conceptuales y metodológicos por lo que realmente ocurre en los procesos de enseñanza y aprendizaje de la historia en las aulas, la pregunta de investigación que guía esta investigación exploratoria es ¿Cuáles son las características, formatos y contenidos de los cuadernos escolares de Historia de México e Historia Universal de una cohorte documental?

Objetivos: Explorar con la técnica de análisis documental de sistematización y análisis de contenido una cohorte de cuadernos escolares de historia.

\section{Metodología}

Para contextualizar esta fuente de investigación el primer paso fue recuperar el mayor número posible de evidencias (cuadernos escolares) en distintos grados y niveles educativos por lo que se constituyó en una investigación educativa a partir de los siguientes parámetros: el análisis y valoración de los cuadernos de primaria en la que el contenido de historia se relaciona con el de otras asignaturas y el análisis y valoración de los cuadernos de secundaria en el que el contenido de historia se agrupa en un cuaderno particular.

En 2017, la Universidad Pedagógica Nacional, en su Unidad 151-Toluca, inició la línea de investigación sobre cuadernos escolares de historia que inscribe en el campo la 
cultura escrita y la educación histórica. El proceso de investigación culminó dos años después con la conformación de la colección digital de cuadernos de historia CESHIS que implicó dos momentos o fases de investigación: El primero fue la conformación de la base de datos de los cuadernos de historia que incluyó las actividades de a) acopio de cuadernos de Historia de primaria y secundaria; b) fichas y captura de datos; c) digitalización de los cuadernos de historia y en el segundo momento el diseño de la colección digital de acceso abierto.

\section{Fase 1: El acopio de cuadernos de Historia de primaria y secundaria.}

La estrategia de acopio de los cuadernos escolares se realizó al finalizar el ciclo escolar 2017, lo que permitió integrar una primera cohorte de los cuadernos de Historia de México e Historia Universal de los ciclos escolares 2011-2016. En los primeros meses del 2018 se integró una segunda cohorte de cuadernos escolares que marca el término del modelo curricular de la RIEB.

Es importante señalar la dificultad que representó hacer el acopio de los cuadernos de historia que los niños elaboraron en los ciclos escolares del inicio de la reforma integral, dado que el cuaderno de clase del niño es rápidamente desechado por la familia al terminar cada año escolar. La destrucción a la que se someten estos productos escolares hace que los esfuerzos del investigador por recuperar estas evidencias puedan fracasar, lo que obliga a fortalecer los vínculos con maestros y padres de familia para contar con una estrategia de comunicación efectiva.

Afortunadamente esta investigación contó con el apoyo de alumnos egresados de la maestría en enseñanza de las humanidades y de educación básica de nuestra institución que actualmente se desempeñan como maestros frente a grupo, quienes, de manera generosa, auxiliaron en esta fase de la investigación. En los cuadros siguientes se concentra la información sobre el total de cuadernos de historia de primaria y secundaria recuperados y el desglose por nivel, grado y ciclo escolar. La fase de acopio finalmente tuvo éxito al recuperar un total de setenta y cinco cuadernos de historia de educación básica distribuidos de la siguiente manera (Tabla 1): 


\begin{tabular}{cc}
\hline Nivel & Cuadernos \\
\hline Primaria & 27 \\
Secundaria & 48 \\
Total & 75 \\
\hline
\end{tabular}

Tabla 1. Acopio de cuadernos de Historia. Fuente: Base de datos de cuadernos escolares.

El siguiente paso fue agrupar el total de cuadernos de Historia por nivel (primaria y secundaria) grado y asignatura, lo que permitió identificar también los ciclos escolares que se cubren en la base de datos. Se elaboró una ficha de registro para cada uno de los cuadernos de Historia que incluye los datos del alumno, el ciclo escolar, la asignatura y el grado.

Los cuadernos de Historia de primaria se clasificaron por grado y asignatura lo que permitió que se cubrieran los cuatro grados en los que se imparte la asignatura de historia en la educación primaria: en tercer grado el cuaderno aparece con el nombre de la asignatura "Conocimiento del medio", en cuarto y quinto grado con el contenido de Historia de México y finalmente los de sexto grado de Historia Universal.

Los cuadernos de cuarto, quinto y sexto grado cubren el ciclo completo de inicio y cierre de la reforma integral de educación básica 2011-2016. Esta característica permite documentar los cambios y las permanencias en los formatos y contenidos de las asignaturas Historia de México e Historia Universal. Los cuadernos de tercer grado en el que se imparte la historia en el campo de conocimiento del medio, solamente permiten documentar el periodo de cierre de la reforma integral en el ciclo escolar 2015 - 2016 (Tabla 2). 
Tabla 2. Acopio de cuadernos de Historia de primaria por grado. Fuente: Base de datos de cuadernos escolares.

\begin{tabular}{llcc}
\hline Grado & Asignatura & $\begin{array}{l}\text { Ciclos } \\
\text { escolares }\end{array}$ & No. cuadernos \\
\hline Tercero & Conocimiento del medio & $2015-2016$ & 2 \\
Cuarto & Historia de México & $2010-2011$ & 8 \\
& & $2014-2015$ & \\
Quinto & Historia de México & $2012-2013$ & 6 \\
& & $2014-2015$ & \\
Sexto & Historia Universal & $2016-2017$ & \\
& & $2011-2012$ & 11 \\
& & $2014-2013$ & \\
& & $2015-2016$ & \\
Total & & $2016-2017$ & 27 \\
\hline
\end{tabular}

Los cuadernos de historia de secundaria que se incorporan a esta base de datos ascienden a cuarenta y ocho mismos que fueron clasificados por grado $\left(2^{\circ} \mathrm{o} 3^{\circ}\right)$, por asignatura (Historia de México o Historia Universal) y ciclo escolar, es de señalar que su distribución permite documentar el periodo 2011- 2017 que marcan el inicio y cierre de la reforma de secundaria y su relación con la educación primaria (Tabla 3).

\begin{tabular}{lccc}
\hline Grado & Asignatura & Ciclos escolares & No. cuadernos \\
\hline Segundo & Historia Universal & $2014-2015$ & 18 \\
& & $2015-2016$ & \\
& $2016-2017$ & 30 \\
Tercero & Historia de México & $2010-2011$ & \\
& & $2012-2013$ & \\
& $2014-2015$ & 48 \\
Total & $2016-2017$ & \\
\hline Tabla 3. Acopio de cuadernos de Historia de secundaria por grado. Fuente: Base de datos \\
de cuadernos escolares.
\end{tabular}

El registro de cuadernos de historia de secundaria constituye una muestra muy importante pues recordemos que en este nivel la asignatura es impartida lo mismo por profesores con formación en historia, como por aquellos que provienen de otros campos de conocimiento. El importante número de casos de cuadernos de historia de secundaria permite documentar 
ampliamente la enseñanza y el aprendizaje de la Historia de México e Historia Universal desde el 2011 hasta el 2017. Esto cobra particular importancia pues es, en la escuela secundaria, donde se condensan los propósitos centrales del aprendizaje de la historia: la formación del pensamiento crítico y analítico del alumno de secundaria.

Fase 2: La base de datos y la ficha de contenido.

La base de datos recupera los siguientes elementos: a) los formatos b) las actividades y c) la organización del contenido disciplinar:

a) Los formatos. Incluye las imágenes de los formatos y marcas de los alumnos en la "carátula", se presentan los datos que permiten identificar: escuela, profesor, alumno, etc. Las carátulas, separadores por bloques, y marcas de organización de las actividades: el "tiempo", el tipo de "actividad" y el "contenido disciplinar". El "tiempo" es el primer eje a partir del cual se ordenan los cuadernos y está dividido en meses y días.

b) Las actividades o ejercicios, la organización general del cuaderno, la fecha del ejercicio o actividad. El cuaderno se ordena en función de las actividades y la numeración por lo cual se hacen calculables. Es posible también identificar el tipo de actividad que se despliega en el aula: inicial, de desarrollo, final, evaluativa etc.

c) El "contenido disciplinar". La división por disciplinas y temas está realizada al interior de cada ejercicio, mediante indicaciones tales como título y subtítulo. Los temas y las disciplinas tienen igual valor en los cuadernos de clase; dicho valor es el de ser un ejercicio en el total de ejercicios anuales (Tabla 4).

\begin{tabular}{ll}
\hline \multicolumn{1}{c}{ Categorías } & \multicolumn{1}{c}{ Variables } \\
Formatos y actividades didácticas & 1. Características de los formatos, portadas y \\
& carátulas. \\
& 2. Organización del contenido histórico \\
& 3. Marcas del maestro \\
& 4. Marcas del alumno \\
& 5. Líneas del tiempo \\
& 6. Mapas históricos \\
& 7. Esquemas y mapas mentales \\
& 8. Imágenes \\
& 9. Cuestionarios \\
10. Función del dibujo & \multicolumn{1}{c}{ Revista RedCA octubre 2020-enero 2021 } \\
1.BSN: 2594-2824 Vol. 3 Núm. 8
\end{tabular}




\begin{tabular}{ll} 
& 2.Bimestres \\
& 3. Temarios \\
& 4. Procesos \\
& 5.Acontecimientos \\
& 6.Cambios \\
Habilidades del pensamiento & 1. Ubicación del tiempo y el espacio. \\
histórico & 2. Vocabulario histórico \\
& 3. Resúmenes de textos \\
& 4. Uso de fuentes históricas \\
& 5. Uso de copias y lectura de fragmentos \\
& 6. Reflexiones y opiniones \\
& 7.Evaluación \\
\hline
\end{tabular}

Tabla 4. Categorías y variables en el diseño de la base de dato. Elaboración propia.

Estas variables fueron construidas a partir de la revisión minuciosa de los cuadernos de Historia en la primera fase de acopio y se afinaron a partir en la segunda fase de acopio de acopio, ello con la finalidad de adecuar los formatos que sirvieron para la captura de la información cuantitativa y cualitativa.

Fase 3. Digitalización de los cuadernos de Historia de primaria y secundaria

Los cuadernos de historia de primaria y secundaria se empezaron a digitalizar por orden de aparición, lo que permitió revisar y adecuar las variables consideradas en la base de datos y la elaboración de una ficha de contenido. En esta fase, se completaron las fichas de un total de treinta y cinco cuadernos historia de $\operatorname{los}$ grados $4^{\circ}, 5^{\circ}$ y $6^{\circ}$ de primaria y $2^{\circ}$ y $3^{\circ}$ de secundaria.

Se digitalizaron los cuadernos completos desde su portada hasta la contraportada, en un formato de registro de imagen que puede ser editada para su consulta. El total de imágenes digitalizadas de los cuadernos completos dado su número y diversidad permiten aplicar los procedimientos de análisis y valoración de los cuadernos escolares como fuentes de investigación.

\section{Resultados}

Dos productos documentales son resultado de esta investigación: La colección digital de cuadernos de Historia de México e Historia Universal y la base de datos de cuadernos escolares de historia de primaria y secundaria (2011-2017).

Revista RedCA octubre 2020 - enero 2021 ISSN: 2594-2824 Vol. 3 Núm. 8 
La plataforma CESHIS. Colección digital de cuadernos escolares de Historia.

La colección digital de acceso abierto tiene como propósito poner a disposición de los estudiantes e investigadores de México, América Latina y Europa, interesados en la investigación de cuadernos escolares, con la posibilidad de generar trabajos comparativos y en un tiempo corto alimentar las cohortes de cuadernos escolares que documenten las políticas curriculares en nuestro país.

En el siguiente mapa se señala el origen de los cuadernos escolares recuperados en esta plataforma: Mapa de referencia de origen de los cuadernos escolares. (México país, Edo. de México y Ciudad de México) (Figura 1).

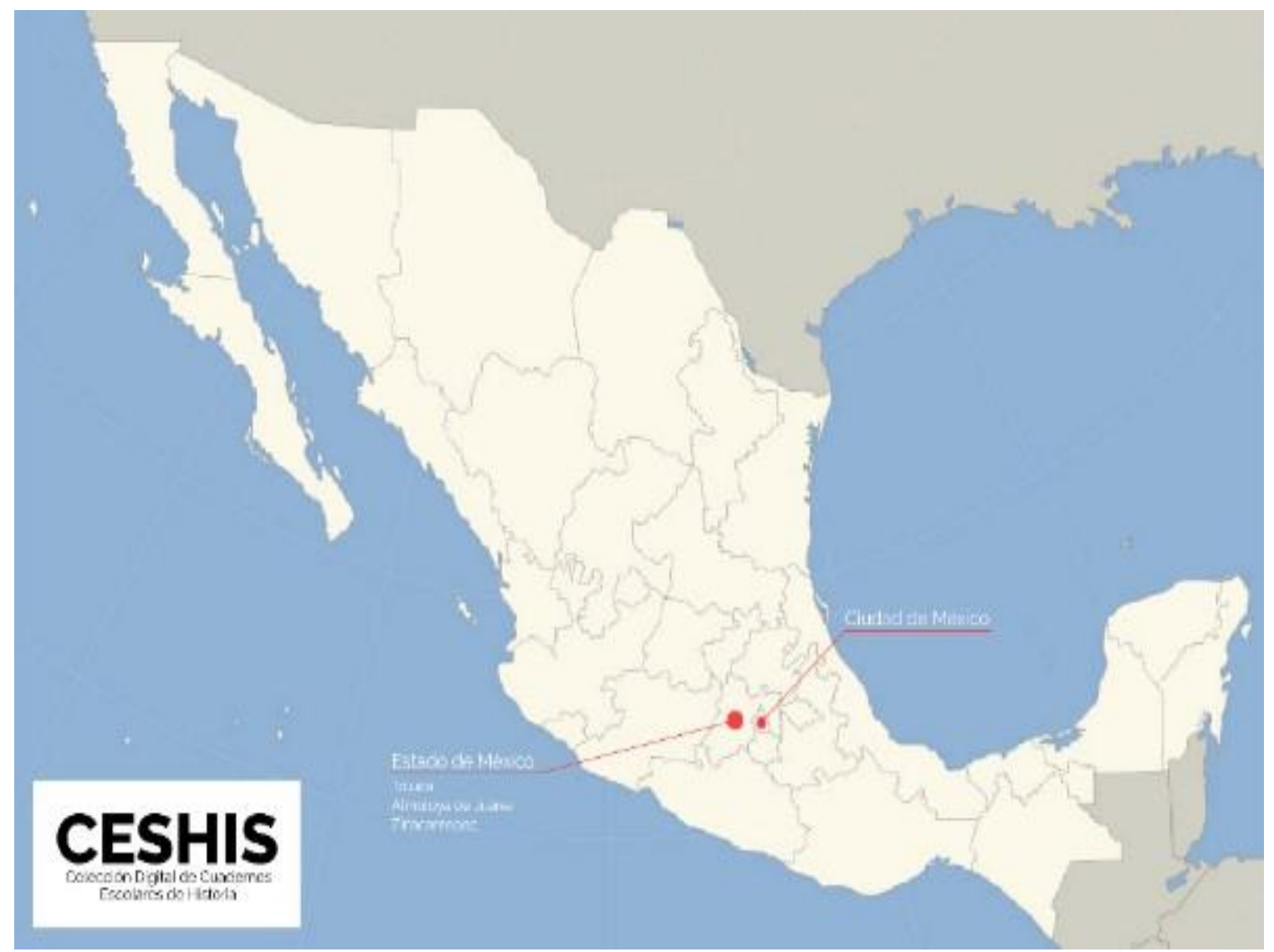

Figura 1. Estados de origen de los cuadernos escolares. 
La colección de historia de México contiene diez cuadernos escolares de 4o. y $5^{\circ}$ grado de primaria y $3^{\circ}$ grado de secundaria; de acuerdo a la RIEB 2011 con esta asignatura se pretende forjar en los estudiantes la noción de tiempo histórico, la comprensión de la multicausalidad y fomentar la identidad nacional a través del conocimiento de los procesos históricos nacionales. La colección se identifica con el nombre propio del alumno, se ubica el año y el lugar de procedencia (Figura 2).

\title{
Cuadernos de Historia de México
}

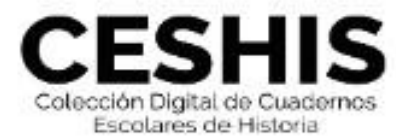

\section{Primaria}

$\begin{array}{ll}\text { Ailton } & 2016 \\ \text { Andrea } & 2014 \\ \text { Jéssica Leslie } & 2012 \\ \text { Karla Denise } & 2012 \\ \text { Laura } & 2014 \\ \text { Mariana } & 2014\end{array}$

Almoloya de Juárez, Estado de México

Toluca, Estado de México

Toluca, Estado de México

Toluca, Estado de México

Toluca, Estado de México

Toluca, Estado de México

\section{Secundaria}

$\begin{array}{ll}\text { Alejandra } & 2015 \\ \text { Andrea } & 2016 \\ \text { Deborah } & 2015 \\ \text { Sofia } & 2016\end{array}$

\author{
Toluca, Estado de México \\ Zinacantepec, Estado de México \\ Toluca, Estado de México \\ Toluca. Estado de México
}

Figura 2. Municipios de origen del Estado de México de los cuadernos escolares.

En esta colección se encontraron 26 cuadernos de historia universal para $6^{\circ}$ grado de primaria y $2^{\mathbf{o}}$ grado de secundaria donde las nociones de tiempo-espacio, cambio, simultaneidad y el desarrollo de una consciencia histórica deberán afianzarse en los alumnos. La colección digital la integran once cuadernos de Historia Universal de sexto grado de primaria y segundo trece de secundaria. La colección de cuadernos escolares de historia universal se inserta en el contexto de la Reforma Integral de Educación Básica (RIEB) 2011 en México donde la enseñanza de la historia pretende desarrollar habilidades cognitivas que 
fomenten en los estudiantes el pensamiento histórico. Los datos se muestran abajo (Figura $3)$.

\section{Cuadernos de Historia universal}

\section{Primaria}

$\begin{array}{ll}\text { Frida } & 2016 \\ \text { Luz Valeria } & 2012 \\ \text { Maria del Pilar } & 2016 \\ \text { Mariana } & 2016 \\ \text { Melisa } & 2016 \\ \text { Monserrath } & 2016 \\ \text { Alejandra } & 2016 \\ \text { Ingrid } & 2016 \\ \text { Jéssica Leslie } & 2013 \\ \text { Josué } & 2016 \\ \text { Juan Alberto } & 2016\end{array}$

\section{Secundaria}

$\begin{array}{ll}\text { Alexis } & 2016 \\ \text { Andrea } & 2015 \\ \text { Bibiana } & 2016 \\ \text { Briza } & 2016 \\ \text { Daniel } & 2016 \\ \text { Fabiola } & 2016 \\ \text { Gisele } & 2016 \\ \text { Jéssica Leslie } & 2015 \\ \text { Maryfer } & 2016 \\ \text { Monserrath } & 2015 \\ \text { Paola } & 2016 \\ \text { Ricardo } & 2012 \\ \text { Vianka } & 2016\end{array}$

\author{
Toluca, Estado de México \\ Toluca, Estado de México \\ Zinacantepec, Estado de México \\ Toluca, Estado de México \\ Ciudad de México \\ Toluca, Estado de México \\ Ciudad de México \\ Ciudad de México \\ Toluca, Estado de México \\ Toluca, Estado de México \\ Toluca, Estado de México
}

Toluca, Estado de México
Toluca, Estado de México
Toluca, Estado de México
Zinacantepec, Estado de México
Toluca, Estado de México
Zinacantepec, Estado de México
Toluca, Estado de México
Toluca, Estado de México
s/d
Toluca, Estado de México
Ciudad de México
s/d
Toluca, Estado de México

Figura 3. Nombres personales y municipio de residencia de los alumnos que donaron sus cuadernos escolares.

El acercamiento a los cuadernos de Historia Universal de los alumnos de primaria y secundaria permite identificar los problemas en la ubicación del tiempo y el espacio 
múltiples, pues es en este grado cuando inicia el acercamiento a las civilizaciones de oriente y el desarrollo de las culturas europeas y su vinculación con las americanas. En estos cuadernos se documentan ampliamente las actividades y un conjunto de ejercicios como; elaboración de mapas históricos, líneas del tiempo etc. tareas que permiten construir las nociones temporales y espaciales en los niños de sexto grado. Los contenidos persiguen que los estudiantes tengan contacto con conceptos y nociones de la disciplina histórica para acercarse a la tarea/actividad histórica. Por ello la enseñanza/aprendizaje de la Historia exige un trabajo continuo con actividades vinculadas a las nociones temporales y espaciales (SEP, 2011)

\section{La base de datos especializada}

El diseño y elaboración de esta base de datos especializada logró recuperar un corpus de 75 cuadernos escolares de Historia de México e Historia Universal de primaria y secundaria que cubren el periodo de 2011- 2017. Su riqueza y diversidad de contenido (actividades, habilidades, formatos) constituye el punto de partida para profundizar en las problemáticas de la enseñanza, pero sobre todo del aprendizaje de la historia en educación básica en contexto de la reforma integral.

Las evidencias recuperadas documentan las características y composición de los escritos que los niños y los adolescentes desarrollan en las aulas; las actividades de lectura y escritura de la historia y las habilidades que se desarrollan con ellas. Hasta ahora, estas características de los cuadernos escolares no habían sido sistematizadas en nuestro país.

A la pregunta sobre si los cuadernos escolares muestran signos de cambio en la construcción de nuevas prácticas que erradiquen las prácticas tradicionales que son herencia del positivismo como los cuestionarios, los resúmenes y el contenido tematizado y fragmentado a lo largo del cuaderno escolar, tenemos todavía una respuesta tentativa. De una muestra de once cuadernos de historia de sexto grado, a la que le aplicamos procedimientos de análisis de contenido, podemos concluir lo siguiente:

Si comparamos las actividades registradas en la siguiente gráfica observamos que las actividades preponderantes siguen siendo la evaluación del maestro para controlar lo escrito por el alumno. Esta es una acción docente que se puede constatar en cada uno de los 
cuadernos escolares revisados. El ejemplo que se presenta, se inscribe en una tipología que privilegia el control sobre la actividad y la necesidad imperiosa de dejar marcas del maestro para evaluar cada uno de los productos del alumno. Esta característica marca una continuidad en las formas tradicionales que tienen un fuerte arraigo en la cultura escolar. Esta huella permite valorar el peso de la evaluación como productora de prácticas discursivas que sin duda están fuertemente ancladas en la concepción de la enseñanza como una medición del conocimiento histórico escolar.

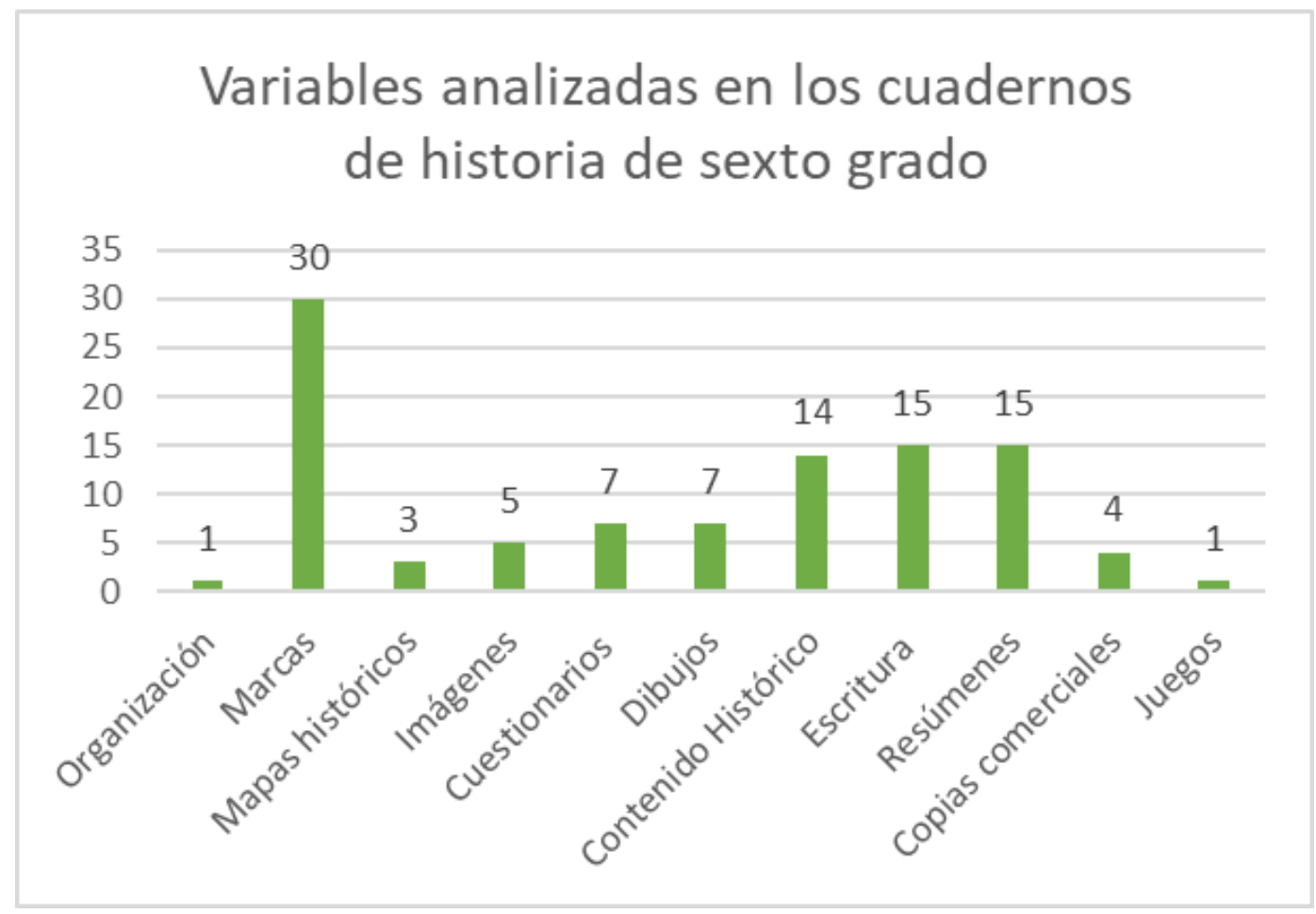

Tabla 5. Variables de análisis de contenido de los cuadernos de Historia. Fuente: Base de datos de cuadernos escolares. CESHIS.

Las actividades marginales en los cuadernos escolares. Un resultado significativo es que el uso de fuentes históricas es una actividad marginal en las aulas de nuestros estudiantes de educación básica como se puede observar en el siguiente ejemplo, donde predomina el uso de cuestionarios y el resumen del contenido históricos escolar. Este tipo de cuaderno es un claro ejemplo del predominio de actividades identificadas con la historia positivista y el uso de las preguntas cerradas que tienen como eje central el desarrollo de la memorización de fechas y datos inconexos. 
Las habilidades desarrolladas vs. habilidades requeridas. En la sistematización de las actividades de lectura de comprensión y lectura crítica de textos, así como la elaboración de escritos en las que se exprese la opinión de los niños y adolescentes, constatamos que las evidencias son limitadas. Está lejos el desarrollo de las nociones de tiempo y espacio histórico $\mathrm{y}$, sobre todo, la posibilidad de que la historia se le represente al niño bajo un esquema de interrogantes que cobren sentido con la realidad que vive actualmente. Sin embargo, hay indicios de que se está desarrollando la observación como una estrategia que permite al niño asociar por ejemplo imágenes y contenido/ desprender algunas inferencias e interrogantes.

Los cuadernos de sexto grado de primaria y sobre todo de secundaria presentan una mayor cantidad de mapas mentales y conceptuales lo que podría estar generando una forma de ordenar y analizar la información histórica. La regularidad con la que aparece el uso de ordenadores de información para el aprendizaje de la historia es signo de los cambios en las prácticas de lectura y análisis de la información. Este hallazgo representa una estrategia que ha sido recomendada por la pedagogía constructivista desde hace algunos años y que se relaciona con los procesos de construcción del conocimiento histórico a partir de los distintos niveles educativos. Es decir, se traduce en un proceso de lectura que tendría sus bases en la escuela primaria para avanzar en procesos de análisis de textos en la escuela secundaria.

Sin embargo, para profundizar en el tipo de prácticas de lectura que se desarrolla en las aulas es necesario comparar y analizar estos gráficos con base en los hallazgos investigativos en torno a los ordenadores y el aprendizaje. Díaz Barriga (1999) hace una tipología de ordenadores gráficos que incluye diversos niveles de asociación de información, elaboración de inferencias y coherencia de los elementos recuperados por el alumno. En los ejemplos recuperados para sexto grado de primaria se identifican elementos de asociación primaria y los conectores analíticos son escasos.

Un rasgo igualmente preocupante es la ausencia en los cuadernos escolares de escritos de reflexión y opinión de los alumnos frente a la lectura de textos de divulgación, libros de texto de historia y documentos de apoyo. Si recordamos que el propósito central de la enseñanza de la historia es elaborar preguntas que generen los propios alumnos sobre su entorno social y los problemas actuales, a partir de las cuales puedan indagar y analizar la 
información de los libros de texto, los libros de divulgación histórica y a la que pueden tener acceso por las redes sociales y el uso de las nuevas tecnologías de la información. Este tipo de actividades que generan la opinión y reflexión de los alumnos se ve restringida y cuando aparece la evidencia la registra en pequeños párrafos. El cuaderno escolar es un instrumento fundamental para "acercarnos a los tiempos" (ritmos, secuencias, momentos) reales de la actividad escolar. Los cuadernos de clase develan el discurso escolar: la tarea docente; la (s) cultura (s), los saberes, los contenidos y las disciplinas escolares.

\section{Conclusiones}

La práctica escolar de la historia ha tenido un desarrollo vigoroso con momentos críticos, entre los que sobresalen los intentos oficiales de reducir su presencia en las aulas, incluso su desaparición so pretexto de una educación que atienda lo "útil" y "concreto" para la vida del ciudadano y el modo de producción en boga. Por suerte, la acción magisterial y el esfuerzo de la llamada sociedad civil, amén de los actos de resistencia de pequeños pero influyentes grupos académicos en las escuelas, han generado una sinergia para mantener viva la historia y mover los hilos en pos de su enseñanza en las aulas, siempre con visiones renovadas.

Por lo que respecta a la investigación histórica, ésta registra momentos importantes y una madurez sin precedentes. La actividad en las universidades y centros de investigación reconoce una elaboración e intentos por generar la memoria de la riqueza de los eventos en que como grupos y sociedad se han concretado.

Los protagonistas de la historia han sido los héroes nacionales, muchos de ellos mitificados y petrificado en monumentos diseñados para la "eternidad"; empero, el surgimiento de los sujetos sociales de la vida cotidiana, abre un nuevo espacio para integrar las visiones y relatos de lo acontecido, de los sucesos y de la concreción de actos que son parte de la vida de los grupos y de las generaciones.

Generar en el campo de la historiografía los insumos para una nueva mirada a la escuela, sus producciones infantiles y las prácticas de escritura en la enseñanza de la historia, es sin lugar a dudas una veta poco explorada, y que por sí misma se vuelve una acción, como 
caja de tiempo, que va acumulando vestigios del acontecer en las aulas sobre la enseñanza de la historia.

Los cuadernos escolares se convierten en una puerta al conocimiento de los sujetos principales en el proceso de enseñanza de la historia: el alumno y el docente. El primero como aprendiz y enseñante de su quehacer como historiador en ciernes, en la compilación de las actividades escolares relacionadas con la materia, organizando sus productos en su cuaderno de trabajo, que da cuenta de las tareas y momentos de acercamiento al aprendizaje de la historia que se muestra en el aula. El segundo también como aprendiz y enseñante de la historia, que juega a sintetizar los rasgos de las formas de actuar para la comprensión entendimiento del tiempo histórico, que permiten alimentar la conciencia del alumno y la suya propia de los hechos del pasado y su incidencia en el presente.

Respecto de la pregunta de investigación, ¿Cuáles son las características, formatos y contenido de los cuadernos escolares de Historia de México e Historia Universal de una cohorte documental?, debe advertirse que los cuadernos contienen sus configuraciones, imbuidas por los estilos docentes de los profesores que dirigen la enseñanza, y muestran un contenido mediado por el plan y programas de estudio, junto con un estilo ya marcado por el proceso educativo implementado en el aula.

En el caso de la exploración con la técnica de análisis documental de sistematización y análisis de contenido una cohorte de cuadernos escolares de historia, fue un logro llegar sistematizar en contenido en un grupo importante de categorías, que reflejan finalmente un interés docente por la evaluación de sus estudiantes, y de éstos, la necesidad de "mostrar" sus conocimientos escolares de la historia, vía el instrumento en el que quedan convertidos los cuadernos. Aunque ello no es la fundamental en los procesos de enseñanza de la historia, sino la formación de una conciencia histórica y un pensamiento crítico, lo cierto es que permite considerar replantear fuertemente las estrategias didácticas que se están utilizando en el aula y considerar nuevas para llegar a tales metas. 


\section{Referencias bibliográficas}

Britto García, L. (2005). Tareas escolares. Educere, 9(30), 405-406. Recuperado de https://www.redalyc.org/pdf/356/35603017.pdf

Carretero, M. (2007). Documentos de Identidad. La construcción de la memoria histórica en el mundo global. Buenos Aires: Paidós.

Cuesta, R. (octubre, 2000). Didáctica crítica y educación histórica. Pensar, desear y actuar de otra manera. Cuadernos de Pedagogía, (295) 62-67. Recuperado de https://redined.mecd.gob.es/xmlui/handle/11162/38301

Cucuzza, H. R. (dir.) y Pineau, P. (coord.) (2002). Para una historia de la enseñanza de la lectura y la escritura en la Argentina. Buenos Aires: Miño y Dávila Editores.

Chartier, A-M. (2005). Los cuadernos escolares: ordenar los saberes escribiéndolos. Conferencia pronunciada en el VIII Congreso Internacional de la cultura escrita. Universidad de Alcalá, 5-8 julio 2005.

Díaz Barriga, F. (1999). Estrategias docentes para un aprendizaje significativo. México: McGraw Hill.

Escolano Benito, A. (2007). La cultura material de la escuela. En A. Escolano Benito (Ed.), La cultura material de la escuela. En el centenario de la JAE, (1907-2007) (pp. 1527). España: CEINCE.

Fernández, I. (2012). Saberes y prácticas escolares. Espacios en Blanco. Revista de Educación, 22, 304-309. Recuperado de https://www.redalyc.org/pdf/3845/384539804016.pdf

Finocchio, S. (2013). Un tesoro inexplorado: los periódicos escolares en la Argentina. Revista História da Educação, 17(40), 27-54. Recuperado de https://www.redalyc.org/pdf/3216/321627379003.pdf 
(2010). Una cartografía de saberes escolares en movimiento para América latina. Propuesta Educativa, (34), 65-76. Recuperado de https://www.redalyc.org/pdf/4030/403041705007.pdf (2005). La ciudadanía en los cuadernos de clase. Enseñanza de las Ciencias

Sociales, $\quad$ (4), 3-10. $\quad$ Recuperado de https://www.redalyc.org/pdf/3241/324127616002.pdf

Fischman, G. (2006). Las fotos escolares como analizadores en la investigación educativa. Educação \& Realidade, 31(2), 79-94. Recuperado de https://www.redalyc.org/pdf/3172/317227044006.pdf

Fraguela-Vale, R., \& Pose-Porto, H., \& Varela-Garrote, L. (2016). Tiempos escolares y lectura. Ocnos: Revista de Estudios sobre Lectura, 15(2), 67-76. Recuperado de https://revista.uclm.es/index.php/ocnos/article/view/ocnos_2016.15.2.1099

Gvirtz, S. (1997). Del currículum prescrito al curriculum enseñado. Una mirada a los cuadernos de clase. Argentina: Aique.

(1998). Análisis del cuaderno como texto. Primera Parte. Revista Novedades Educativas. Buenos Aires-México, 10(96).

Gvirtz, S., Larrondo, M. (s/f). El cuaderno de clase como fuente primaria de investigación. Alcances y límites teóricos y metodológicos para su abordaje. Recuperado de https://www.academia.edu/2571412/El_cuaderno_de_clase_como_fuente_primaria _de_investigaci\%C3\%B3n_Alcances_y_1\%C3\%ADmites_en_la_construcci\%C3\% B3n del objeto de estudio En School Exercise Books A Complex Source for _a_History_of_the_Approach_to_Schooling_and_Education_in_the_19th_and_20th _Centuries_Tomo_I_Ed_Polistampa_Macerata_Italia

Hurtado Beltrán, M. (2011). El papel de los textos escolares en ciencias sociales. Revista Geográfica de América Central, 2, 1-18. Recuperado de https://www.revistas.una.ac.cr/index.php/geografica/article/view/2883 
Peredo Merlo, M. (2001). Las habilidades de lectura y la escolaridad. Perfiles Educativos, 23(94), 57-69. Recuperado de https://www.redalyc.org/pdf/132/13209404.pdf

Postigo, C., \& Lambrisca, B., \& Hernández, C., \& Checa, L. (2004). La producción simbólica en los discursos narrativos escritos de los escolares. En Cuadernos de la Facultad de Humanidades y Ciencias Sociales - Universidad Nacional de Jujuy, (22), 33-45. Recuperado de https://www.redalyc.org/pdf/185/18502203.pdf

Sanchidrián Blanco, C., \& Arias Gómez, B. (2013). La construcción de la identidad nacional española a través de los cuadernos escolares (1957-1959). Revista de Investigación Educativa, 31(1), 257-274. Recuperado de http://revistas.um.es/rie/article/view/148661

Sanchidrián, B. \& Gallegos, Ma. (2003) Los cuadernos escolares como fuente y tema de investigación en Historia de la Educación. España: Universidad de Málaga.

SEP. (2011). Plan de Estudios, Educación Básica. México: SEP.

Viñao Frago, A. (2008). La escuela y la escolaridad como objetos históricos. Facetas y problemas de la historia de la educación. Revista História da Educação, 12(25), 954. Recuperado de http://www.redalyc.org/articulo.oa?id=321627131002

(2007). Los cuadernos escolares como fuente histórica: Aspectos Metodológicos e Historiográficos. Revista Memoria, Conocimiento y Utopía. Sociedad Mexicana de Historia de la Educación (3), 93-105. 